\title{
Context-evoked overperception in asthma
}

\author{
STEVEN DE PEUTER ${ }^{1}$, CLAUDIA PUT ${ }^{2}$, \\ VALENTINE LEMAIGRE ${ }^{2}$, MAURITS DEMEDTS ${ }^{2}$, \\ GEERT VERLEDEN ${ }^{2}$, \& OMER VAN DEN BERGH ${ }^{1}$
}

${ }^{1}$ Department of Psychology, University of Leuven, Tiensestraat 102, B-3000 Leuven, Belgium and ${ }^{2}$ Department of Respiratory Medicine, University Hospital Gasthuisberg, Herestraat 49, B-3000 Leuven, Belgium

(Received 3 March 2006; in final form 13 September 2006)

\begin{abstract}
We investigated associative learning as a possible explanation of overperception in asthma. Thirty newly diagnosed patients with asthma (eight men) underwent a histamine provocation to elicit airway obstruction (Cockcroft's protocol). Patients testing positive and reporting symptoms underwent an identical procedure on the next day with saline, a substance that does not elicit any significant airway changes. Symptoms of airway obstruction and fatigue increased significantly from pre- to post-saline inhalation on day 2, whereas physiological parameters did not change. Increases in symptoms were significantly related to the level of dispositional negative affectivity, but not to the degree of airway reactivity or changes in objective lung function. These results suggest that the experience of asthmatic symptoms in a specific context may evoke the experience of similar symptoms when confronted with the context only. Learning was more likely to occur in those patients with high negative affectivity.
\end{abstract}

Keywords: Asthma, conditioning, histamine, negative affectivity, emotions, symptoms, symptom perception

\section{Introduction}

Asthma is a chronic disease characterized by recurrent attacks of breathlessness, wheezing, and cough due to episodes of airway obstruction. The accuracy of symptom perception in asthma is defined in terms of the relationship

Correspondence: Steven De Peuter, PhD, Department of Psychology, Tiensestraat 102, B-3000 Leuven, Belgium. Tel:. +32 1632.59.23. Fax: +32 1632.59.23. E-mail: steven.depeuter@ psy.kuleuven.be 
between decreases in respiratory function and feelings of breathlessness (Lehrer, Feldman, Giardino, Song, \& Schmaling, 2002). A common problem in clinical practice is that a large proportion of patients reports symptoms in excess of abnormalities in lung function or ventilatory variables (Takano, Inaishi, \& Zhang, 1997). This overperception of symptoms is associated with excessive medication intake, unwarranted illness behavior, hospitalization, and negative emotions (Put, Van den Bergh, Demedts, \& Verleden, 2000). Gender, age, disease severity (Bijl-Hofland, Cloosterman, Folgering, Akkermans, \& Van Schayck, 1999; Bijl-Hofland et al., 1999), the speed of attack onset (Turcotte \& Boulet, 1993), and diurnal variation (Peiffer, Marsac, \& Lockhart, 1989) have been identified as physiological determinants of poor perception. However, also inter-individual differences, probably interacting with other variables such as mood changes and specific personality traits, appear to modulate the accuracy of symptom perception (Chetta et al., 1998), as do psychological factors (e.g., suggestion, attentional and interpretative processes, and illness schemata; De Peuter et al., 2004; Lehrer et al., 2002).

Recently, De Peuter et al. showed that associative learning may also influence symptom perception in asthma (De Peuter et al., 2005). Framed as a test of "a new chemical for diagnosing asthma", a placebo inhaler was associated three times with breathing $\mathrm{CO}_{2}$-enriched air for $2 \mathrm{~min}$, whereas a control placebo inhaler was associated three times with room air breathing. The results from the subsequent test phase without $\mathrm{CO}_{2}$ suggested that associative learning effects were responsible for experimentally induced overperception: Participants reported elevated symptoms in response to the placebo inhaler that had been paired with $\mathrm{CO}_{2}$-enriched air. These elevated symptom reports occurred without accompanying changes in physiological responses.

However, $\mathrm{CO}_{2}$-enriched air inhalation elicits mild and transient symptoms dominated by an increase in ventilation, but it does not actually induce bronchoconstriction. As such, although asthma-like symptoms were reported in the De Peuter et al. study, $\mathrm{CO}_{2}$-enriched air inhalation has only limited ecological validity as a respiratory challenge to investigate symptom perception in asthma.

A replication of these results in a setting where real bronchoconstriction is induced would provide strong support for the relevance of learning for overperception in asthma. We decided to take advantage of the standardized histamine provocation used to diagnose new patients with asthma. During this provocation, patients inhale histamine, triggering bronchoconstriction. We considered the experience of symptoms of actual bronchoconstriction in this specific context as a learning experience and we hypothesized that participants returning the next day for the same procedure without histamine (inhaling only a neutral substance, in this case saline) would report an increase of respiratory symptoms, without an accompanying airway response.

We also expected that overperception would be most pronounced in participants characterized by high neuroticism or "negative affectivity" (NA; the tendency to experience negative emotions, together with a negative selfimage, introspectiveness, and dwelling on failures and shortcomings...; Costa \& 
McCrae, 1987; Pennebaker 1982, 2000; Watson \& Clark, 1984). In asthma, this variable is associated with enhanced symptom-reporting: Previously hospitalized high-NA asthmatic out-patients reported as much respiratory symptoms after discharge from the hospital as during their hospitalization, despite radical improvement in lung function and daily functioning (Put, Demedts, Van den Bergh, Demyttenaere, \& Verleden, 1999). According to Main, Moss-Morris, Booth, Kaptein and Kolbe (2003) this may be because NA leads patients to associate a greater number of symptoms with their asthma, including nonasthma symptoms such as signs of physiological arousal. In that respect, patients with high NA have more opportunities to learn associations between (what they consider) asthma symptoms and contextual cues.

\section{Methods}

\section{Participants}

Patients who were referred to the pulmonary function testing unit of the university hospital for a histamine provocation were invited to participate in the study. Inclusion criteria were: (1) asthma diagnosis according to American Thoracic Society criteria (ATS, 1991), including a positive histamine challenge test; (2) no symptoms after baseline saline inhalation on day 1 and respiratory symptoms after histamine inhalation on day 1 (see procedure section); (3) age between 18 and 60 . On study days, participants had not taken any medication that protected them from the bronchoconstrictive effect of histamine. This was stressed when the appointment was made and checked verbally by the laboratory assistant before both provocations.

\section{Subjective measures}

We used the Asthma Symptom Checklist (ASC; Kinsman, Luparello, O'Banion, \& Spector, 1973; Ritz, Bobb, Edwards, \& Steptoe, 2001) to assess subjective asthma symptoms. Its 36 items fall into six subscales: symptoms of airway obstruction (e.g., chest congestion, chest tightening), dyspnea (e.g., hard to breathe, short of breath), symptoms suggestive of hyperventilation (e.g., dizzy, tingling in spots), fatigue (e.g., tired, no energy), anxiety (e.g., frightened, worried about attack), and irritation (e.g., nervous, irritable). Internal consistency for five subscales is high (Cronbach's $\alpha$ : ranging from 0.86 to 0.93 ) and acceptable for the hyperventilation subscale (Cronbach's $\alpha$ : 0.76; Put, 2001). Participants indicated on an 11-point scale the currently experienced intensity of symptoms $(0=$ no symptoms, $10=$ symptoms as bad as possible).

To measure negative emotionality, we used the 14-item abbreviated StressReaction subscale from Tellegen's Brief Multidimensional Personality Questionnaire (Patrick, Curtin, \& Tellegen, 2002). Scores range from 0 (low NA) to 14 (high NA). High scorers describe themselves as nervous, apprehensive, irritable, overly sensitive, and emotionally labile. Because the Stress-Reaction subscale contains no somatic symptoms or health-related items, it is particularly 
suited for health research (Watson, 1988). Internal consistency is high (Patrick et al., 2002).

\section{Respiratory physiology}

As a measure of airway hyperreactivity, we determined the provocative concentration of histamine required to produce a $20 \%$ fall in forced expiratory volume in one second ( $\mathrm{PC}_{20}$ hist; see the subsequent text). All patients underwent spirometry according to European Respiratory Society guidelines (Quanjer et al., 1993) to determine forced expiratory volume in one second $\left(\mathrm{FEV}_{1}\right)$ and forced vital capacity (FVC). We also determined airway resistance (ResFR; Ritz et al., 2002). Values obtained from spirometry and the measurement of airway resistance are indications of the status of the airways, and were used to distinguish physiological reactivity to the saline inhalations from learning effects.

\section{Procedure}

On day 1, patients underwent a standardized histamine provocation (Cockcroft's protocol; Cockcroft, Killian, Mellon, \& Hargreave, 1977). Baseline spirometry was performed, immediately followed by a measurement of baseline respiratory resistance. Then, patients inhaled saline $(\mathrm{NaCl} ; 0.9 \%)$, followed by progressive doubling concentrations of histamine $\left(0.25-8 \mathrm{mg} \mathrm{mL}^{-1}\right)$ until the $\mathrm{FEV}_{1}$ had fallen at least $20 \%$ below baseline values or until the maximum concentration of $8 \mathrm{mg} \mathrm{mL}^{-1}$ was reached. The histamine dose required to elicit a fall in lung function larger than $20 \%$ of the baseline value ( $\mathrm{PC}_{20}$ hist) is indicative of the degree of airway hyperresponsiveness, lower doses suggesting higher (hyper)reactivity. Immediately after each inhalation respiratory resistance was measured; spirometry was repeated 1 and $3 \mathrm{~min}$ after each inhalation. The participants were told that the procedure investigated the influence of inhaling certain substances on respiratory function and subjective symptoms, but they were not informed about the nature of the substances they were inhaling or the gradual increase of dosages. Participants testing positive $\left(\mathrm{PC}_{20}\right.$ hist $\left.<8 \mathrm{mg} \mathrm{mL}^{-1}\right)$ and reporting complaints during the provocation test returned the next day for "a second part of the test". They underwent a similar procedure as on the first day, but they inhaled four times saline $(0.9 \% \mathrm{NaCl})$ and no histamine. The inhalation of saline may elicit a minimal effect on respiratory function in a subgroup of patients, but in general there are no effects.

The Stress-Reaction scale was completed on the first day; the ASC was filled out pre- and post-test on both days. At the end of the procedure on the second day, participants were fully debriefed.

All participants provided informed consent and the Medical Ethical Board of the University Hospital approved the study.

\section{Data analysis}

Per measure moment (pre-histamine provocation, post-histamine provocation, pre-saline provocation, post-saline provocation) means were calculated for the 
six symptom subscales and the physiological parameters. A median split of the Stress-Reaction scores divided the participants in a high and low NA group. For each inhalation condition separately (histamine versus saline), two repeated measures ANOVA's were performed: one for the physiological parameters with one within subject variable (measure moment: pre versus post); another for ASC-subscale scores with one within subject variable (measure moment) and one between subject variable (NA). Computational analyses were performed with the statistical program STATVIEW for Windows (1999, SAS Institute Inc, USA).

\section{Results}

\section{Demographics and clinical characteristics}

Thirty patients with asthma ( 8 men, 22 women; mean age 38 years $(S D=16)$; range 18-58) participated in the study (Table I). None of the patients had a respiratory infection at the time of the study, and lung function data were within the normal range. An average $\mathrm{PC}_{20}$ hist of $2.7 \mathrm{mg} \mathrm{mL}^{-1}(\mathrm{SD}=1.9)$ suggests most participants had mild asthma. Nine participants had at least one positive rast test for a common allergen (pollen, housedustmite, cat, dog etc.). At the moment of testing, none of the participants were taking oral or intravenous steroids. Six participants used inhaled steroids, and five of them combined this with $\beta_{2}$-agonists or anticholinergics. The mean Stress-Reaction score was 3.9; a median split at 4.0 divided the group in high $(M \pm S D=6 \pm 1)$ and low $(M \pm \mathrm{SD}=0.9 \pm 1) \mathrm{NA}$ participants. All patients were naive to the histamine challenge test.

Table I. Demographics and clinical characteristics of the patients with asthma.

\begin{tabular}{lc}
\hline Descriptive parameter & \\
\hline Demographics & \\
Age & $38 \pm 16$ \\
Gender (m/f) & $8 / 22$ \\
Physiological parameters & \\
FEV $(\%$ pred) & $104 \pm 17$ \\
FVC (\% pred) & $107 \pm 16$ \\
ResFR (Hz) & $11.5 \pm 3.9$ \\
PC ${ }_{20}$ hist (mg mL ${ }^{-1}$ ) & $2.7 \pm 1.9$ \\
Medication intake (\%) & \\
Inhaled steroids & 20 \\
$\beta_{2}$-agonists (S.A.) & 27 \\
$\beta_{2}$-agonists (L.A.) & 0 \\
Anticholinergics & 16 \\
\hline
\end{tabular}

Notes: \% pred, percentage of predicted value, S.A., short-acting; L.A., long-acting. 
Table II. The influence of the histamine and saline provocations on respiratory parameters and asthma symptoms.

\begin{tabular}{|c|c|c|c|c|c|c|}
\hline & \multicolumn{3}{|c|}{ Histamine inhalation } & \multicolumn{3}{|c|}{ Saline inhalation } \\
\hline & pre & post & $F$-values ${ }^{\mathrm{a}}$ & pre & post & $F$-values ${ }^{\mathrm{a}}$ \\
\hline $\mathrm{FEV}_{1}(\mathrm{~L})$ & $3.3 \pm 0.9$ & $2.4 \pm 0.6^{\star}$ & 99.5 & $3.2 \pm 0.9$ & $3.2 \pm 0.8$ & n.s. \\
\hline $\mathrm{FVC}(\mathrm{L})$ & $4.1 \pm 1.1$ & $3.5 \pm 0.9^{\star}$ & 110.9 & $4.0 \pm 1.1$ & $4.0 \pm 1.1$ & n.s. \\
\hline ResFR & $11.5 \pm 3.9$ & $28.8 \pm 8.5^{\star}$ & 110.9 & $11.7 \pm 5$ & $12.7 \pm 5.9$ & n.s. \\
\hline OBS & $1.1 \pm 1.4$ & $3.0 \pm 2.0^{\star}$ & 43 & $0.7 \pm 0.9$ & $1.3 \pm 1.5^{\dagger}$ & $6.2^{\mathrm{b}}$ \\
\hline DYS & $1.6 \pm 2.2$ & $4.9 \pm 2.3^{\star}$ & 64.2 & $1.0 \pm 1.2$ & $1.5 \pm 1.8$ & n.s. ${ }^{\mathrm{b}}$ \\
\hline FAT & $1.1 \pm 1.5$ & $2.1 \pm 2.2^{\star}$ & 10.8 & $0.9 \pm 1.1$ & $1.3 \pm 1.6^{t}$ & $4.8^{\mathrm{b}}$ \\
\hline HYP & $0.3 \pm 0.5$ & $0.8 \pm 0.8^{\dagger}$ & 9 & $0.3 \pm 0.3$ & $0.4 \pm 0.6$ & n.s. ${ }^{b}$ \\
\hline IRR & $0.6 \pm 1.2$ & $1.0 \pm 1.6^{\dagger}$ & 7.7 & $0.4 \pm 0.8$ & $0.4 \pm 0.9$ & n.s. ${ }^{b}$ \\
\hline ANX & $0.3 \pm 0.5$ & $0.8 \pm 1.6^{\star}$ & 4.6 & $0.2 \pm 0.4$ & $0.3 \pm 0.7$ & n.s. ${ }^{b}$ \\
\hline
\end{tabular}

Notes: Difference with pre test: ${ }^{\star} p<0.0001 ;{ }^{\dagger} p<0.01 ;{ }^{\star} p<0.05$; ANX, Anxiety subscale of the ASC; DYS, Dyspnea subscale of the ASC; FAT, Fatigue subscale of the ASC; HYP, hyperventilation subscale of the ASC; IRR, Irritability subscale of the ASC; OBS, obstruction subscale of the ASC.

${ }^{\mathrm{a}} F(1,29)$ for $\mathrm{FEV}_{1}, \mathrm{FVC}$ and ResFR; $F(1,28)$ for ASC subscales.

$\mathrm{b}_{n=27,3}$ outliers were left out.

\section{Histamine provocation}

On average $( \pm S D)$ there were $4.7 \pm 1.6$ histamine inhalations per participant. For all respiratory parameters there was a significant difference between pre- and post-histamine challenge (Table II). The average changes were $-27 \%,-14 \%$, and $150 \%$ for $\mathrm{FEV}_{1}, \mathrm{FVC}$ and ResFR, respectively (for $F$-values, see Table II). All ASC subscale scores increased significantly from pre- to post-histamine inhalation (Table II).

High NA participants reported more symptoms than low NA-participants for hyperventilation complaints, irritability, and anxiety (NA main effects; $F(1,28)=5.6,4.7$, and 5.7 , respectively; all $p$ 's $<0.05)$. Only the anxiety scores increased significantly more in high than in low NA participants between pre- and post-histamine inhalation (NA $\times$ Measure moment interaction; $F(1,28)=4.4$, $p<0.05)$. The increase in dyspnea scores between pre- and post-histamine inhalation was related to the decrease in $\mathrm{FEV}_{1}$ between pre- and post-inhalation $(r=0.37, p<0.05)$, but not to $\mathrm{PC}_{20}$ hist, NA, or changes in FVC or ResFR ( $r$ 's between 0.08 and 0.24 , all nonsignificant). The increase in obstruction scores was unrelated to these parameters ( $r$ 's between 0.12 and 0.22 , all nonsignificant).

\section{Saline 'provocation'}

All the physiological parameters showed no difference between pre and post 4 times saline inhalation (Table II). The average change was $-1 \%$ (range from -13 to $12 \%$ ), $-0.7 \%$ (range from -8 to $11 \%$ ), and $11 \%$ (range from -20 to $195 \%$ ), for $\mathrm{FEV}_{1}, \mathrm{FVC}$, and ResFR, respectively. In three patients (9\% of the sample) ResFR increased more than $50 \%$. When these three cases were 


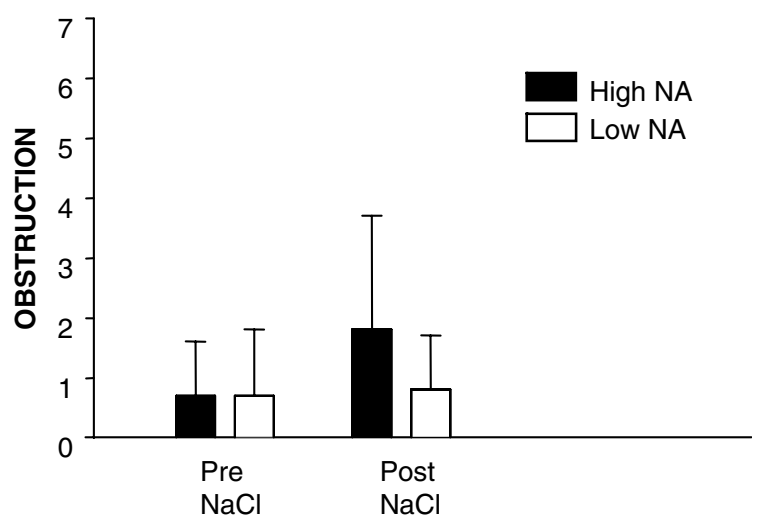

Figure 1. NA-interaction effect for symptoms of obstruction for the saline 'provocation'.

excluded from the analysis, the average change for ResFR became $-2 \%$ (range from -20 to $18 \%$ ).

Symptom scores of obstruction and fatigue increased significantly from pre to post 4 times saline inhalation, $F(1,30)=10.9$ and 6, respectively; $p<0.005$ and $<0.05$, respectively. The pre- to post-saline increases in obstruction and fatigue symptoms remained significant when the three patients with airway reactions to saline inhalation were excluded (Table II). Across the total sample, the increase in obstruction scores correlated with an increase in $\operatorname{ResFR}(r=0.50$, $p<0.01$ ).

High NA participants reported more irritability than low NA participants (NA main effect; $F(1,27)=11.4, p<0.01)$. The increase in obstruction scores from pre to post 4 times saline inhalation was present in high NA participants, but not in low NA participants (NA $\times$ Measure moment interaction, Figure 1; $F(1,27)=4.5, p<0.05)$.

Differences between pre and post 4 times saline inhalation obstruction and fatigue scores were significantly related to NA $(r=0.51, p<0.01 ; r=-0.47$, $p<0.01$, respectively), but not to $\mathrm{PC}_{20}$ hist, or changes in $\mathrm{FEV}_{1}, \mathrm{FVC}$, or ResFR. NA was unrelated to lung function ( $r$ 's between 0.08 and 0.26 , all nonsignificant).

\section{Discussion}

We investigated the influence of an experience of histamine-induced bronchoconstriction on subsequent symptom-reporting in the same context in patients with mild asthma. All patients experienced a decrease of lung function values and an increase in asthma symptoms in the specific context of a standardized histamine provocation. Increases in feelings of dyspnea (items: hard to breathe, short of breath, wheezing) were associated with decreases in lung function, whereas symptoms of airway obstruction (items: chest congestion, chest 
tightening, chest filling up, chest pain, uncomfortable) were unrelated to respiratory parameters.

Repeating the procedure on the following day with saline instead of histamine caused a significant increase of symptoms of airway obstruction without accompanying decreases in lung function. Differences in airway reactivity could not explain the symptoms either.

This increase in symptoms of airway obstruction was specific for high NA participants. Although fatigue also increased, this effect did not interact with NA. It is interesting to see that the effects were most pronounced for high NA subjects. In the study of De Peuter and colleagues (2005), there was no effect of NA on learning. They interpreted this as an effect of the context of their experiment, suggesting that attention may have increased in both high and low NA participants, consequently erasing habitual attentional differences between the groups.

High NA subjects have been shown to be more vigilant to respiratory sensations (attentional bias) and worry more about the sensations (negative interpretational bias) in comparison with low NA subjects (Stegen et al., 1998, 1999). However, differences in vigilance between high and low NA persons may not show up in conditions without threat, while in conditions with clear threat both types of persons may direct attention to the threat source (Martin, Rothrock, Leventhal, \& Leventhal, 2003). In mildly threatening conditions, individual differences may show up because high NA persons may tag the situation as more threatening and/ or because the low NA subjects are inclined to avoid rather trivial aversive events (see also Mogg \& Bradley, 1998). For patients newly diagnosed with asthma, the situation of a histamine provocation is new, induces relatively distinct symptoms, and is likely to draw both high and low NA patients' attention equally. This may explain why, on the first day of the study, high NA participants in the present study did not report more typical respiratory symptoms (symptoms of dyspnea and obstruction) than low NA patients. Nevertheless, they showed stronger emotional responses overall (symptoms of hyperventilation and irritability) and their anxiety increased more from pre- to post-histamine. The saline inhalations on the following day provided them with a familiar context and more ambiguous sensations, creating an opportunity for memory information triggered by the contextual cues to influence the interpretation of these sensations as symptoms.

The effect of NA from the present study is consistent with the findings of Main et al. (2003) that asthma patients with high NA tend to interpret (i.e., mislabel) somatic sensations as symptoms of asthma. These results are also in line with the "concept of symmetry" from Leventhal's Common Sense Model of Illness (Martin et al., 2003): Patients high in NA reported symptoms that they believe are consistent with the diagnostic label (or "prototype", see Bishop, 1991), which they "borrow" from the histamine provocation on the previous day.

Although our effects were reliable, they were modest in size, possibly because of the relatively weak manipulation: The standardized histamine provocation uses a limited number of trials, provoking relatively minor airway responses and mild subjective symptoms. Repeatedly occurring symptom episodes of a much stronger 
nature, such as naturally occurring asthma exacerbations, may result in stronger effects because they are associated with more severe airway obstruction and hence more symptoms and more arousal. Because asthma attacks are mostly unpredictable and patients do not have the reassuring presence of a physician as they did in the hospital, they will probably search the environment even harder for predictive cues. As the attacks become represented in memory, subsequent experiences sharing common elements with the represented information may automatically trigger memory codes that help structure (i.e., bias) the actual experience. This tendency to use situational information in symptom perception is pronounced in asthma patients (Rietveld \& Houtveen, 2004) and the perception of asthma patients typically deteriorates with time due to this process of selective perception (Rietveld \& Brosschot, 1999; Rietveld \& Prins, 1998).

One could suggest that our results can also be explained by social desirability or the fact that repeated inhalations of saline elicit symptoms of obstruction. If demand effects caused the results, we would expect increased symptom reports both for symptoms of dyspnea and obstruction (as these symptoms increased after inhalation of histamine). This was not the case. In addition, we assessed respiratory symptoms verbally after the saline inhalation on day 1 , and participants did not report any respiratory symptoms at that time. For the majority of the participants, inhaling saline did not elicit changes in respiratory functioning. Removing those patients with an airway reaction to saline from the analyses did not alter the results. Moreover, the fact that the learning effect was specific for high NA patients counters the explanation in terms of saline-induced symptoms.

The present study has some limitations. First, we did not vary contextual cues among groups and we did not test for mere repetition effects by including a group with saline inhalations on both days. Nevertheless, our findings are consistent with earlier findings showing that asthma symptom reporting can be learned in well-controlled laboratory conditions (De Peuter et al., 2005). Moreover, our results extends those findings in that we used a situation as associative cue (rather than a single cue such as a placebo inhaler) and actual bronchoconstriction instead of $\mathrm{CO}_{2}$-enriched air inhalation. This increases the ecological validity of the current experiment compared to the former study, in that the gradual induction of actual bronchoconstriction bears much closer resemblance to a real-life attack situation than inhaling "a chemical" for 2 min. Second, although saline is relatively broncho-inert, airway resistance increased by $>50 \%$ in three participants and their symptom reporting after saline inhalation was related to these physiological changes. Nevertheless, removing these participants from the analyses did not alter the main results of this study. Third, our sample was rather small, possibly reducing the power of the statistical tests. Future studies need larger samples to identify effects with other symptoms.

These results have important clinical implications: Because asthma patients rely mainly on perceived symptoms and feelings of 'discomfort' for their medication use (Nouwen, Freeston, Cournoyer, Deschesnes, \& Boulet, 1994) it is likely that they will take reliever medication based on expected symptoms 
instead of real exacerbations of respiratory dysfunction (De Peuter et al., 2005). Therefore, it is important to help patients to be as accurate as possible in the assessment of their respiratory functioning. The use of peak flow meters to train patients to recognize signs of obstruction has been suggested before. Clinicians also need to rely on objective measures rather than subjective symptom reports when evaluating disease severity and prescribing medication. Our results also suggest the importance of identifying individuals high in NA in primary practice, as these patients will be most vulnerable to learn incorrect associations. An explicit assessment of the triggers that patients associate with their asthma can be used to correct erroneous beliefs.

In conclusion, the induction of respiratory symptoms by histamine in patients with asthma subsequently leads to similar symptoms of obstruction when patients are confronted with the same context, without the bronchoconstricting agent being administered. This effect of prior symptom experience was particularly observed in subjects characterized by high negative affectivity. Experimental manipulations involving more trials with more intense respiratory challenges, mimicking real life asthma symptoms closer would probably enhance the ecological validity of the present approach, but may not be ethically warranted.

\section{Acknowledgments}

The first author (S.D.P.) was supported during the study by grant G.0270.01 (FWO-Flanders) and BIL01/05 of the Bilateral Scientific and Technological Cooperation between Flanders (Belgium) and China; and currently by PDM/05/ 075 from the KULeuven. The second author (C.P.) was supported by grant 7.0004.000 (FWO-Flanders) and Astra Pharmaceuticals Belgium.

\section{References}

Bijl-Hofland, I. D., Cloosterman, S. G. M., Folgering, H. Th. M., Akkermans, R. P., \& van Schayck, C. P. (1999). Relation of the perception of airway obstruction to the severity of asthma. Thorax, 54, 15-19.

Bijl-Hofland, I. D., Folgering, H. Th. M., van den Hoogen, H., Cloosterman, S. G. M., Van Weel, C., Donkers, J. M., et al. (1999). Perception of bronchoconstriction in asthma patients measured during histamine challenge test. European Respiratory Fournal, 14, 1049-1054.

Bishop, G. D. (1991). Understanding the understanding of illness: Lay disease representations. In J. A. Skelton \& R. T. Croyle (Eds), Mental representations in health and illness (pp. 32-59). New York: Springer New York.

Chetta, A., Gerra, G., Foresi, A., Zaimovic, A., Del Donno, M., Chittolini, B., et al. (1998). Personality profiles and breathlessness perception in outpatients with different gradings of asthma. American Fournal of Respiratory and Critical Care Medicine, 157, 116-122.

Cockcroft, D. W., Killian, D. N., Mellon, J. J., \& Hargreave, F. E. (1977). Bronchial reactivity to inhaled histamine: A method and clinical survey. Clinical Allergy, 7, 235-243.

Costa, P. T., \& McCrae, R. R. (1987). Neuroticism, somatic complaints and disease: Is the bark worse than the bite? Fournal of Personality, 55, 299-316.

De Peuter, S., Van Diest, I., Lemaigre, V., Verleden, G., Demedts, M., \& Van den Bergh, O. (2004). Dyspnea: The role of psychological processes. Clinical Psychology Review, 24, 557-581. 
De Peuter, S., Van Diest, I., Lemaigre, V., Wan, L., Verleden, G., Demedts, M., et al. (2005). Can subjective asthma symptoms be learned? Psychosomatic Medicine, 67, 454-461.

Kinsman, R. A., Luparello, T., O’Banion, K., \& Spector, S. (1973). Multidimensional analysis of the subjective symptomatology of asthma. Psychosomatic Medicine, 35, 250-267.

Lehrer, P., Feldman, J., Giardino, N., Song, H.-S., \& Schmaling, K. (2002). Psychological aspects of asthma. Fournal of Consulting and Clinical Psychology, 70, 691-711.

Main, J., Moss-Morris, R., Booth, R., Kaptein, A. A., \& Kolbe, J. (2003). The use of reliever medication in asthma: The role of negative mood and symptom reports. Fournal of Asthma, 40, 357-365.

Martin, R., Rothrock, N., Leventhal, H., \& Leventhal, E. (2003). Common sense models of illness: Implications for symptom perception and health-related behaviors. In J. Suls \& K. A. Wallston (Eds), Social psychological foundations of health and illness (pp. 199-225). Malden: Blackwell Publishing Ltd.

Mogg, K., \& Bradley, B. P. (1998). A cognitive-motivational analysis of anxiety. Behaviour Research and Therapy, 36, 809-848.

Nouwen, A., Freeston, M. H., Cournoyer, I., Deschesnes, F., \& Boulet, L.-P. (1994). Perceived symptoms and discomfort during induced bronchospasm: The role of temporal adaptation and anxiety. Behaviour Research and Therapy, 32, 623-628.

Patrick, C. J., Curtin, J. J., \& Tellegen, A. (2002). Development and validation of a brief form of the Multidimensional Personality Questionnaire. Psychological Assessment, 14, 150-163.

Peiffer, C., Marsac, J., \& Lockhart, A. (1989). Chronobiological study of the relationship between dyspnoea and airway obstruction in symptomatic asthmatic subjects. Clinical Science (Colch), 77, 237-244.

Pennebaker, J. W. (1982). The psychology of physical symptoms. New York: Springer-Verlag.

Pennebaker, J. W. (2000). Psychological factors influencing the reporting of physical symptoms. In A. A. Stone, J. S. Turkkan, C. A. Bachrach, J. B. Jobe, H. S. Kurtzman, \& V. S. Cain (Eds), The science of self-report. Implications for research and practice (pp. 299-315). London: Lawrence Erlbaum Associates.

Put, C. (2001). Unpublished doctoral dissertation. In Symptom perception in asthma. Medicine meets psychology. Belgium: University of Leuven.

Put, C., Van den Bergh, O., Demedts, M., \& Verleden, G. (2000). A study of the relationship among self-reported noncompliance, symptomatology, and psychological variables in patients with asthma. Fournal of Asthma, 37, 503-510.

Put, C., Demedts, M., Van den Bergh, O., Demyttenaere, K., \& Verleden, G. (1999). Asthma symptoms: Influence of personality versus clinical status. European Respiratory fournal, 13, 751-756.

Quanjer, P. H., Tammeling, G. J., Cotes, J. E., Pedersen, O. F., Peslin, R., \& Yernault, J. C. (1993). Lung volumes and forced ventilatory flows. Report Working Party Standardization of Lung Function Tests, European Community for Steel and Coal. Official Statement of the European Respiratory Society. European Respiratory fournal, 6, 5-40.

Rietveld, S., \& Brosschot, J. F. (1999). Current perspectives on symptom perception in asthma: A biomedical and psychological review. International fournal of Behavioral Medicine, 6, 120-134.

Rietveld, S., \& Houtveen, J. H. (2004). Acquired sensitivity to relevant physiological activity in patients with chronic health problems. Behaviour Research and Therapy, 42, 137-153.

Rietveld, S., \& Prins, P. J. M. (1998). Children's perception of physical symptoms. The example of asthma. Advances in Clinical Child Psychology, 20, 153-182.

Ritz, T., Bobb, C., Edwards, M., \& Steptoe, A. (2001). The structure of a symptom report in asthma: A reevaluation. Fournal of Psychosomatic Research, 51, 639-645.

Ritz, T., Dahme, B., DuBois, A. B., Folgering, H., Fritz, G. K., Harver, A., et al. (2002). Guidelines for mechanical lung function measurements in psychophysiology. Psychophysiology, $39,546-567$. 
Stegen, K., Van Diest, I., Van de Woestijne, K. P., \& Van den Bergh, O. (1999). Negative affectivity and bodily sensations induced by $5.5 \% \mathrm{CO}_{2}$ enriched air inhalation: The role of interpretative processes. Psychology and Health, 15, 1-13.

Stegen, K., Neujens, A., Crombez, G., Hermans, D., Van de Woestijne, K. P., \& Van den Bergh, O. (1998). Negative affect, respiratory reactivity, and somatic complaints in a C02 enriched air inhalation paradigm. Biological Psychology, 49, 109-122.

Takano, N., Inaishi, S., \& Zhang, Y. Z. (1997). Individual differences in breathlessness during exercise, as related to ventilatory chemosensitivities in humans. Fournal of Physiology (London), 499, 843-848.

Turcotte, H., \& Boulet, L.-P. (1993). Perception of breathlessness during early and late asthmatic responses. American Review of Respiratory Disease, 148, 514-518.

Watson, D. (1988). Intraindividual and interindividual analyses of positive and negative affect: Their relation to health complaints, perceived stress, and daily life activities. Fournal of Personality and Social Psychology, 54, 1020-1030.

Watson, D., \& Clark, L. A. (1984). Negative Affectivity: The disposition to experience aversive emotional states. Psychological Bulletin, 96, 465-490. 